\title{
Does the Method of Cases Rest on a Mistake?
}

Moti Mizrahi

St. John's University

Forthcoming in Review of Philosophy and Psychology

\begin{abstract}
In this paper, I argue that the method of cases (namely, the method of using intuitive judgments elicited by intuition pumps as evidence for and/or against philosophical theories) is not a reliable method of generating evidence for and/or against philosophical theories. In other words, the method of cases is unlikely to generate accurate judgments more often than not. This is so because, if perception and intuition are analogous in epistemically relevant respects, then using intuition pumps to elicit intuitive judgments is like using illusions to elicit perceptual judgments. In both cases, judgments are made under bad epistemic circumstances.
\end{abstract}

Keywords: illusion; intuition; intuition pump; method of cases; intuitive appearance; intellectual seeming; sensory seeming 


\section{Introduction}

A cursory look at the literature of almost any field in philosophy shows that intuitions play a major role in philosophical theorizing. ${ }^{1}$ Philosophers often use intuitive judgments elicited by intuition pumps as evidence for and/or against philosophical theories. Here is how some philosophers describe the evidential role that intuitions play in philosophical arguments:

A popular strategy in philosophy is a certain sort of thought experiment I call intuition pump (Dennett 1984, p. 12, original emphasis; see also Dennett 1995; cf. Dorbolo 2006).

The rules of the game in this sort of analytic project are relatively clear: any proposed analysis is tested against particular cases, usually imaginary, for which we have strong intuitions. The accuracy with which the judgments of the analysis match the deliverances of intuition then constitutes a measure of the adequacy of the analysis (Maudlin 2007, pp. 146-147).

\footnotetext{
${ }^{1}$ By "intuition" I mean "intellectual seeming." According to Brogaard (forthcoming), intellectual seemings ("it intellectually seems that $p$ ') are "seemings that result from implicit or explicit armchair reasoning, where armchair reasoning is reasoning that involves both a priori principles and past experience." Chudnoff (2011b, p. 626) divides views on the nature of intuitions into two broad categories. According to doxastic views, intuitions are doxastic attitudes or dispositions. See, e.g., Williamson (2004), Williamson (2007), and Sosa (2009). According to perceptualist views, intuitions are "pre-doxastic experiences that [...] represent abstract matters as being a certain way" (Chudnoff 2011b, p. 626). See, e.g., Huemer (2007) and Pryor (2005). According to Chudnoff (2011b, p. 626), "Perceptualist views differ from doxastic views in that according to them intuitions are not identical to doxastic attitudes or doxastic dispositions, but lead to doxastic attitudes and doxastic dispositions when taken at face value" (emphasis added). In other words, on perceptualist views, intuitions are prima facie evidence for beliefs (see Chudnoff 2011a and Huemer 2007). In this paper, I am concerned with the epistemology — not the nature —of intuitions. In particular, I am concerned with the method of cases as a way of generating evidence for and/or against philosophical theories.
} 
It is safe to say that these intuitions — and conclusions based on them - determine the structure of contemporary debates in epistemology, metaphysics, and philosophy of logic, language, and mind. Clearly, it is our standard justificatory procedure to use intuitions as evidence (or as reasons) (Bealer 2000, pp. 2-3).

One thing that distinguishes philosophical methodology from the methodology of the sciences is its extensive and avowed reliance on intuition. Especially when philosophers are engaged in philosophical "analysis", they often get preoccupied with intuitions. [...] The evidential weight accorded to intuition is often very high, in both philosophical practice and philosophical reflection (Goldman 2007, p. 1).

Contemporary analytic philosophy is based upon intuitions. Intuitions serve as the primary evidence in the analysis of knowledge, justified belief, right and wrong action, explanation, rational action, intentionality, consciousness and a host of other properties of philosophical interest. Theories or analyses of the properties in question are attacked and defended largely on the basis of their ability to capture intuitive judgements (Pust 2001, p. 227). 
Appeals to intuition play a foundational role in a good deal of philosophical theory construction (Kornblith 2007, p. 28). ${ }^{2}$

Here are two examples of intuitive judgments elicited by intuition pumps that are then used as premises in philosophical arguments.

Example 1: Jackson's (1982, p. 130) Mary

Mary is a brilliant scientist who is, for whatever reason, forced to investigate the world from a black and white room via a black and white television monitor. She specializes in the neurophysiology of vision and acquires, let us suppose, all the physical information there is to obtain about what goes on when we see ripe tomatoes, or the sky, and use terms like 'red', 'blue', and so on. She discovers, for example, just which wavelength combinations from the sky stimulate the retina, and exactly how this produces via the central nervous system the contraction of the vocal chords and expulsion of air from the lungs that results in the uttering of the sentence 'The sky is blue'... What will happen when Mary is released from her black and white room or is given a color television monitor? Will she learn anything or not? It seems just obvious that she will learn something about the world and our visual experience of it. But then is it inescapable that her previous knowledge was incomplete. But she had all the physical information. Ergo there is more to have than that, and Physicalism is false.

\footnotetext{
${ }^{2}$ It should be noted that, contrary to the philosophers quoted here, Cappelen (2012) argues that intuitions do not play an evidential role in philosophical arguments.
} 
Based on this hypothetical case, Jackson reasons roughly as follows:

(J1) Upon considering the hypothetical case of Mary the neuroscientist, it intellectually seems (or intuitively appears) to me (i.e., Jackson) that Mary learns something new upon her release.

(J2) Therefore, Mary learns something new upon her release.

(J3) If Mary learns something new upon her release, then physicalism is false.

(J4) Therefore, physicalism is false.

\section{Example 2: Thomson's (1971, pp. 48-49) Violinist}

You wake up in the morning and find yourself back to back in bed with an unconscious violinist. A famous unconscious violinist. He has been found to have a fatal kidney ailment, and the Society of Music Lovers has canvassed all the available medical records and found that you alone have the right blood type to help. They have therefore kidnapped you, and last night the violinist's circulatory system was plugged into yours, so that your kidneys can be used to extract poisons from his blood as well as your own. The director of the hospital now tells you, "Look, we're sorry the Society of Music Lovers did this to you-we would never have permitted it if we had known. But still, they did it, and the violinist is 
now plugged into you. To unplug you would be to kill him. But never mind, it's only for nine months. By then he will have recovered from his ailment, and can safely be unplugged from you." Is it morally incumbent on you to accede to this situation? No doubt it would be very nice of you if you did, a great kindness. But do you have to accede to it? What if it were not nine months, but nine years? Or longer still? What if the director of the hospital says, "Tough luck. I agree. But now you've got to stay in bed, with the violinist plugged into you, for the rest of your life. Because remember this. All persons have a right to life, and violinists are persons. Granted you have a right to decide what happens in and to your body, but a person's right to life outweighs your right to decide what happens in and to your body. So you cannot ever be unplugged from him." I imagine you would regard this as outrageous.

Based on this hypothetical case, Thomson reasons roughly as follows:

(T1) Upon considering the hypothetical case of the violinist, it intellectually seems (or intuitively appears) to me (i.e., Thomson) that I have no moral obligation to remain attached to the violinist.

(T2) Therefore, I have no moral obligation to remain attached to the violinist. 
(T3) If I have no moral obligation to remain attached to the violinist, then, by analogy, a pregnant woman has no moral obligation to carry a fetus, which is the result of an unplanned pregnancy, for nine months.

(T4) Therefore, a pregnant woman has no moral obligation to carry a fetus, which is the result of an unplanned pregnancy, for nine months.

Clearly, intuitive judgments elicited by intuition pumps play a major role in these arguments. Whether the source of 'Mary learns something new upon her release' and 'I have no moral obligation to remain attached to the violinist' is properly characterized as intuition or not is beside the point. For present purposes, the important point is that, in both arguments, the moves from (J1) to (J2) and from (T1) to (T2) are based (whether explicitly or implicitly) on the following inference:

(S1) In response to hypothetical case $C$, it intellectually seems (or intuitively appears) to $S$ that $p$.

(S2) Therefore, $p$.

Bach (1984, p. 38) calls this kind of reasoning "default reasoning." According to Bach (1984, p. 43), default reasoning is based on what he calls the "take-for-granted rule": 
(TFG) If it seems to me that $p$, then infer that $p$, provided no reason to the contrary occurs to me.

For example, to Jackson (1982, p. 130), "It seems just obvious that [Mary] will learn something about the world" (emphasis added), so he uses (whether explicitly or implicitly) this intellectual seeming or intuitive appearance as evidence for the claim that Mary will learn something about the world, and that is how he gets premise (J2) in his argument against physicalism. Note that the claims 'It intellectually seems to me that Mary learns something new' and 'It intuitively appears to me that Mary learns something new' are different from the claim 'Mary learns something new'. The first two are reports about how things in the Mary case seem or appear to one, whereas the third is a fact about the Mary case itself. Also note that Jackson says nothing else in support of 'Mary will learn something about the world' other than that "it seems just obvious."

Likewise, to Thomson (1971, p. 49) it seems "outrageous" to say that one has a moral obligation to remain attached to the violinist, so she uses (whether explicitly or implicitly) this intellectual seeming or intuitive appearance as evidence for the claim that one is not morally obliged to remain attached to the violinist, and that is how she gets premise (T2) in her argument against anti-abortion views. Again, note that the claims 'It intellectually seems to me that I am not morally obliged to remain attached to the violinist' and 'It intuitively appears to me that I am not morally obliged to remain attached to the violinist' are different from the claim 'I am not morally obliged to remain attached to the violinist'. The first two are reports about how things in the Violinist case seem or appear to one, whereas the third is a fact about the Violinist case itself. Also note that Thomson says nothing else in support of 'I have no moral obligation to remain 
attached to the violinist' other than "I imagine you would regard ['I am morally obliged to remain attached to the violinist'] as outrageous."

The method of cases, then, consists of two steps:

\section{The Method of Cases (MoC)}

Step 1: Intuition Pump. In this step, $S$ considers a hypothetical case $C$ that is supposed to elicit an intuition (i.e., an intellectual seeming or intuitive appearance) $I$.

Step 2: Appeal to Intuition. In this step, $S$ uses the content of $I$ (whose justifier is that it intellectually seems/intuitively appears to $S$ that $I$ is the case in $C$ ) as a premise in an argument for or against a philosophical theory $T^{3}$

For example, upon considering the Mary case, it intellectually seems (or intuitively appears) to Jackson that Mary learns something new upon her release. Jackson then uses (whether explicitly or implicitly) the content of this intuition (i.e., that Mary learns something new upon her release) as a premise in his argument against physicalism. Likewise, upon considering the violinist case, it intellectually seems (or intuitively appears) to Thomson that she is not morally obligated to remain attached to the violinist. Thomson then uses (whether explicitly or implicitly) the content of this intuition (i.e., that she is not morally obligated to remain attached to the violinist) as a premise in her argument against anti-abortion views.

\footnotetext{
${ }^{3}$ On why appeals to intuition are weak arguments, see Mizrahi (2012) and Mizrahi (2013).
} 
In this paper, I argue that the $\mathrm{MoC}$ is not a reliable method of generating evidence for and/or against philosophical theories. In other words, the $\mathrm{MoC}$ is unlikely to generate accurate judgments more often than not. This is so because, if perception and intuition are analogous in epistemically relevant respects, then using intuition pumps to elicit intuitive judgments is like using illusions to elicit perceptual judgments. Many philosophers, especially those who defend the evidential role of intuitions, endorse the perception-intuition analogy (see, e.g., Bonjour 1998 and Bealer 1998). In what follows, I argue that from the perception-intuition analogy it follows that the MoC is not a reliable method of fixing philosophical belief. ${ }^{4}$ This is an interesting result, I think, because of the ubiquity of the $\mathrm{MoC}$ in philosophy and the fact that the perceptionintuition analogy is usually invoked to support the use of intuitions as evidence in philosophical arguments, not argue against such a use (see, e.g., Hales 2012).

\section{The perception-intuition analogy}

As noted above, several philosophers have argued that intellectual intuition and sense perception are analogous in epistemically relevant respects. For example, according to Sosa (1996, p. 154):

Seemings, then, whether sensory or intellectual, might be viewed as inclinations to believe on the basis of direct experience (sensory) or understanding (intellectual) and regardless of any collateral reasoning, memory, or introspection where the objects of intellectual seeming also present themselves as necessary (original emphasis).

\footnotetext{
${ }^{4}$ In this paper, I am not concerned with the experimentalist challenge to the method of cases. See, e.g., Swain et al (2008), Ludwig (2010), Nagel (2012), and Stich (2012). See also Kuntz and Kuntz (2011). Cf. Buckwalter (2012). Although the experimentalist critique of the use of intuitions as evidence is clearly within the scope of issues concerning the epistemology of intuition, I would like to take a different approach in this paper. The approach is to take the perception-intuition analogy seriously, as those who defend the use of intuitions as evidence do, and then see what follows from that.
} 
Arguably, the most prominent, recent proponent of the perception-intuition analogy is Chudnoff (2011a, 2011b). Most recently, Chudnoff (2013, pp. 362-364) has argued for the following analogy between perceptual knowledge and intuitive knowledge:

Perceptual Knowledge (PK): If a perception makes a belief that $\mathrm{p}$ based on it amount to knowledge, it does so in virtue of (1) being an experience in which it perceptually appears to you that $p$, and (2) being an experience in which you are sensorily aware of an item o, such that (3) o makes p true.

Intuitive Knowledge (IK): If an intuition makes a belief that $\mathrm{p}$ based on it amount to knowledge, it does so in virtue of (1) being an experience in which it intuitively appears to you that $\mathrm{p}$, and (2) being an experience in which you are intellectually aware of an item o, such that (3) o makes p true.

Then Chudnoff (2013, p. 364) adds:

The structural parallels between (IK) and (PK) should be obvious. The differences are that intuitive appearance replaces perceptual appearance and intellectual awareness replaces sensory awareness. 
If Chudnoff is right, then in much the same way that $S$ is justified in believing that $p$, where $p$ is the content of a sensory appearance in virtue of which $S$ is sensorily aware of an object $O$ that makes $p$ true, $S$ is justified in believing that $p$, where $p$ is the content of an intellectual appearance in virtue of which $S$ is intellectually aware of an object $O$ that makes $p$ true. Unlike the concrete objects of perception, however, the objects of intuition are abstract, according to Chudnoff (2013).

Like Chudnoff, Hales (2012) also invokes the perception-intuition analogy in order to defend the epistemic role of rational intuition. According to Hales (2012, p. 180):

there is a faculty of rational intuition that delivers prima facie justified beliefs about philosophical propositions. [...] If anything is a faculty, then sense perception is. If intuition is sufficiently similar to perception, then it too counts as a faculty. Moreover, if perception produces prima facie justified beliefs about its target subject matter and thereby serves as a source of knowledge, then so does intuition.

If Hales is right, then we should treat rational intuition as a source of prima facie justified beliefs in much the same way that we treat perception as a source of prima facie justified beliefs.

In what follows, I grant for the sake of argument that the analogy between sense perception and intellectual intuition holds. The question I am concerned with is the following: Is 'it intuitively appears to $S$ that $p$ ' (or 'it intellectually seems to $S$ that $p$ ') a reliable justifier for $p$ ? 
If intuition is indeed analogous to perception in epistemically relevant respects, then the answer will partially depend on the circumstances in which it intuitively appears to $S$ that $p$. In the case of perception, if it sensorily appears to $S$ that $p$ under bad epistemic circumstances, such as those of paradox illusions, such as the Penrose Tribar (see Figure 1) and the Penrose Stairs (see Figure 2), and optical illusions, such as the Müller-Lyer illusion (see Figure 3) and the Ponzo illusion (see Figure 4), then the sensory appearance that $p$ is not a reliable justifier for $p$.

Figure 1. It appears that the tribar is made of three straight beams of square cross-section that meet pairwise at right angles at the vertices of the triangle they form, which is impossible in three-dimensional space. (Image from Wikimedia Commons:

https://commons.wikimedia.org/wiki/Penrose_triangle).

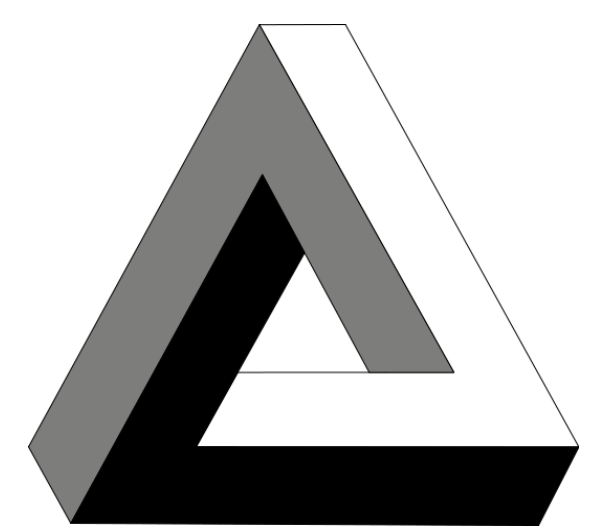


Figure 2. It appears that the stairs make four ninety-degree turns as they ascend or descent and yet they form a continuous loop, such that a person could climb them endlessly, which is impossible in three-dimensional space. (Image from Wikimedia Commons:

https://commons.wikimedia.org/wiki/Penrose_triangle).

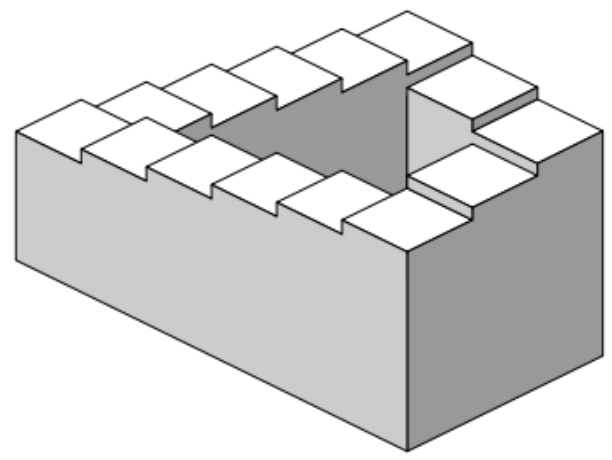

Figure 3. It appears that the line on the right is longer than the line on the left. (Image from Wikimedia Commons: https://commons.wikimedia.org/File:Müller-Lyer_illusion).
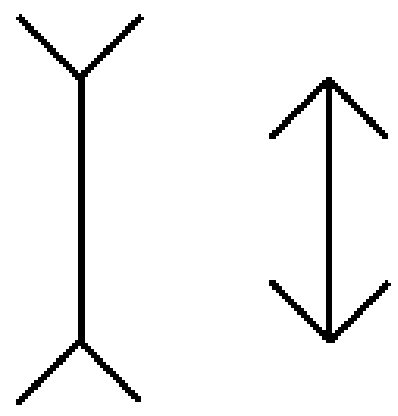
Figure 4. It appears that the top horizontal line is longer than the bottom horizontal line. (Image from Wikimedia Commons: https://commons.wikimedia.org/File:Ponzo_illusion).

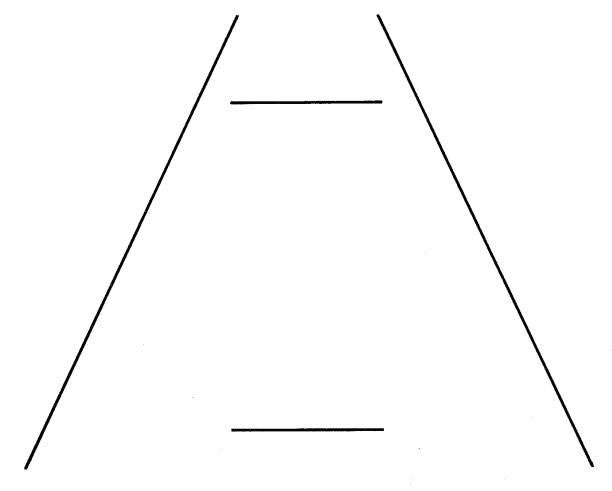

Similarly, in the case of intellectual intuition, if it intuitively appears or intellectually seems to $S$ that $p$ under bad epistemic circumstances, such as those of intuition pumps, then the intuitive appearance or intellectual seeming that $p$ is unreliable as a justifier for $p$. In the next section, then, I argue that intuition pumps are bad epistemic circumstances in much the same way that illusions are.

\section{Bad epistemic circumstances}

If we take the analogy between sense perception and intuition seriously, as those who invoke it in defense of the evidential role of intuitions do, then it follows that the epistemic status of the content of intuitions (as when it intellectually seems to $S$ that $p$ ) and the content of sense 
perceptions (as when it sensorily seems to $S$ that $p$ ) is analogous as well. In that case, it is important to ask whether the circumstances in which intuitive and perceptual judgments are made are epistemically good circumstances. As epistemic circumstances under which judgments are made, intuition pumps are bad epistemic circumstances in much the same way that illusions are, or so I argue. I use the term 'illusion' to refer to images such the Penrose Tribar (see Figure 1) and the Penrose Stairs (see Figure 2), which are known as "paradox illusions," as well as images such as the Müller-Lyer illusion (see Figure 3) and the Ponzo illusion (see Figure 4), which are known as "optical illusions." The first premise of my overall argument, then, is the following:

(1) Making intuitive judgments under bad epistemic circumstances is as unreliable as making perceptual judgments under bad epistemic circumstances.

This follows straightforwardly from the perception-intuition analogy. As further support for (1), however, consider the following. According to one plausible explanation for illusions, like the Penrose Tribar (see Figure 1) and the Penrose Stairs (see Figure 2), such "paradox illusions" arise because what we see clashes with our implicit assumptions about how the world works. For instance, in the case of the Penrose Tribar, we assume that adjacent edges must join, and so it appears that the tribar is made of three straight beams of square cross-section that meet pairwise at right angles at the vertices of the triangle they form, even though that is impossible in threedimensional space. Similarly, in the case of the Penrose Stairs, we assume that the stairs must join, and so it appears that the stairs make four ninety-degree turns as they ascend or descent and 
yet they form a continuous loop, such that a person could climb them endlessly, even though that is impossible in three-dimensional space.

It is important to note that these implicit assumptions do not have to be conscious or in our immediate awareness. Hermann von Helmholtz was probably the first to talk about "unconscious inferences" vis-à-vis illusions (Schett 1999). ${ }^{5}$ Since then, however, the idea has gained much support from studies in perceptual psychology. Several studies suggest that, in forming a coherent picture of the external world, we do make inferences, albeit "unconscious inferences," from sensory input. For example, the rubber hand illusion shows how we "adopt" the rubber hand as our own based on a combination of visual input, i.e., seeing the rubber hand, and tactile input, i.e., having one of our hands stroked with a paintbrush (Botvinick and Cohen 1998; Ehrsson, Holmes, and Passingham 2005). The rubber hand illusion is a form of body transfer illusion (Slater et al 2010).

Similarly, in intuition pumps, a rather similar form of "illusion" arises, I submit, because what we intellectually "see" clashes with our implicit assumptions about the hypothetical case in question. For example, in the Mary case, some, like Jackson, "see" with their mind's eye that Mary learns something new upon her release given that she is supposed to have "complete physical information of color perception." But what does it even mean to have "complete physical information"? Do we really understand this notion any better than the concept of "nonphysical information"? One's judgment about the Mary case, then, will depend on how one understands these notions, namely, "complete physical information" and "non-physical information." That is partly why not everyone shares Jackson's intuition that Mary learns

\footnotetext{
${ }^{5}$ According to Brogaard (forthcoming), intellectual seemings ('it intellectually seems that $p$ ') are "seemings that result from implicit or explicit armchair reasoning, where armchair reasoning is reasoning that involves both a priori principles and past experience" (emphasis added).
} 
something new upon her release (see, e.g., Dennett 1991). So, depending on how one understands the notions of "complete physical information" and "non-physical information," one will intuit that Mary learns something new or that she does not in order to make sense of the Mary case.

Likewise, in the violinist case, some, like Thomson, "see" with their mind's eye that one is not morally obligated to remain attached to the violinist for nine months given that most people cannot afford to be in this situation for nine months. But what if one can afford to be in this situation for nine months? So, depending on one's assumptions about one's own personal circumstances at the time, one will intuit that one has or that one does not have a moral obligation to remain attached to the violinist in order to make sense of the violinist case.

Some might think that intuitive and perceptual judgments actually differ in that respect. That is, even if intuitive judgments are affected by the circumstances of the intuiter (i.e., the intuiter's assumptions, beliefs, abilities, etc.), perceptual judgments are not affected by the circumstances of the perceiver (i.e., the perceiver's assumptions, beliefs, abilities, etc.). Several studies, however, suggest that this is not the case. In other words, perceptual judgments in response to geometrical illusions, such as the Müller-Lyer illusion (see Figure 3), do seem to vary across cultures. To cite one classical study as an example, Segall et al (1963) present data from fifteen societies

showing substantial intersocial differences of two types in susceptibility to geometric optical illusions. The pattern of response differences suggests the existence of different 
habits of perceptual inference which relate to cultural and ecological factors in the visual environment (emphasis added) ${ }^{6}$

As Rookes and Wilson (2000, p. 95) put it, "These findings lend powerful support to the idea that our physical environment can affect our perceptual experience." If sense perception and intuition are indeed analogous, then our intuitions, too, are probably affected by our environment. ${ }^{7}$

Since the work of Stein and Meredith (1993), the notion of multisensory integration has gained much credence. According to Lewkowicz and Ghazanfar (2009):

The objects and events that make up our everyday experience provide us with a constant flow of sensory signals in multiple modalities. Although such inputs can potentially create confusion, our ability to integrate multisensory information enables us to have coherent and meaningful perceptual experiences.

We are fooled by illusions partly because our senses work in conjunction, rather than in isolation, and so sensory input from one sensory modality can interfere and/or override sensory input from another sensory modality. Likewise, since intuition pumps are confusing in much the same way

\footnotetext{
${ }^{6}$ See also Coren and Girgus (1978).

${ }^{7}$ In that respect, perceptions probably vary across cultures in much the same way that intuitions do. On the cultural variation of intuitions, see Machery et al 2012.
} 
that illusions are, insofar as we can be pulled in opposite directions upon considering them (e.g., Mary learns something new or she does not) depending on how the hypothetical cases are presented to us and the implicit assumptions we make about them, it is reasonable to expect that we can be similarly fooled by intuition pumps.

Accordingly, the second premise of my overall argument is the following:

(2) Intuition pumps are bad epistemic circumstances in much the same way that illusions are bad epistemic circumstances.

From (1) and (2) it follows that

(3) Making intuitive judgments in response to intuition pumps is as unreliable as making perceptual judgments in response to illusions.

If this argument is sound, then just as making perceptual judgments under bad epistemic circumstances, such as the circumstances of a perceiver who is faced with an illusion (e.g., the Penrose Tribar), is a recipe for getting things wrong about the image in question more often than not, making intuitive judgments under bad epistemic circumstances, such as the circumstances of an intuiter who is faced with an intuition pump (e.g., the Mary case), is a recipe for getting things wrong about the case in question more often than not. 


\section{Objections and replies}

To sum up, my overall argument runs as follows:

(1) Making intuitive judgments under bad epistemic circumstances is as unreliable as making perceptual judgments under bad epistemic circumstances.

(2) Intuition pumps are bad epistemic circumstances in much the same way that illusions are bad epistemic circumstances.

(3) Therefore, making intuitive judgments in response to intuition pumps is as unreliable as making perceptual judgments in response to illusions.

In Section 3, I provided support for the two premises of this overall argument. In this section, I anticipate and reply to five objections against this argument.

First objection: One might object to my argument by claiming that there is an epistemically relevant difference between intuitive judgments elicited by intuition pumps and perceptual judgments elicited by illusions. More specifically, we can revise our judgments vis-àvis an illusion once we are told it is an illusion. But we cannot easily revise our judgments vis-àvis an intuition pump once we are told it is an intuition pump. In other words, intellectual seemings are more persistent than sensory seemings.

In reply to the first objection, I would like to make two points. First, note that even when we are told that the Penrose Tribar, say, is an illusion it still seems to us that the tribar is made of three straight beams of square cross-section that meet pairwise at right angles at the vertices of the triangle they form. Once we are told that the Penrose Tribar is an illusion, we might be inclined to revise our judgment that tribar is made of three straight beams of square cross-section 
that meet pairwise at right angles at the vertices of the triangle they form. But the content of our sensory seeming is unlikely to change; it is still <the tribar is made of three straight beams of square cross-section that meet pairwise at right angles at the vertices of the triangle they form >, since it still seems to us that the tribar is made of three straight beams of square cross-section that meet pairwise at right angles at the vertices of the triangle they form. As it turns out, the content of this sensory seeming is false. Similarly, when we consider the Mary case, it might seem to us that Mary learns something new. Once we are told that the story about Mary is an intuition pump designed to refute physicalism, we might be inclined to revise our judgment that Mary learns something new. But the content of our intellectual seeming is unlikely to change; it is still <Mary learns something new> (or <Mary does not learn something new $>$ ), since it still seems to us that Mary learns something new (or that she does not).

Second, perceptual judgments elicited by illusions are more persistent than one might think. Consider, for example, the McGurk Effect, which shows that what we see sometimes overrides what we hear (McGurk and MacDonald 1976). Even when one finds out that the McGurk Effect is an illusion, the effect still works. In fact, it works on researchers who have studied it for years. So I think there are no good reasons to believe that intellectual seemings are more persistent than sensory seemings. And, even if they were, it would still not follow that they are epistemically more reliable than sensory seemings.

Second objection: One might also object to my overall argument by claiming that intuition pumps can be distinguished based on their quality (Rescher 2005). If so, then, using some factor to assess the quality of intuition pumps, one could argue that there are epistemically bad intuition pumps, and so we should not put much rational confidence in our intuitions about these intuition pumps, but there are also epistemically good intuition pumps. One such factor, for 
instance, might be the specificity of the hypothetical case. That is to say, the more detailed the hypothetical case in the relevant respects, the better the intuition pump (Brendel 2004).

In reply to the second objection, I would like to make the following points. Recall that what makes illusions epistemically bad circumstances is that what we see clashes with some of our implicit assumptions about the image in question. To make sense of these illusions, then, we "fill in the details," as it were, in a way that makes sense to us. Similarly, what makes intuition pumps epistemically bad circumstances is that what we "see" with the mind's eye clashes with some of our implicit assumptions about the hypothetical case in question. To make sense of these hypothetical cases, then, we "fill in the details," in a way that makes sense to us. The problem is that each person will probably fill in the details in a way that makes sense to him or her and there is no principled way to control for that kind of details-filling when constructing an intuition pump. ${ }^{8}$

For example, in the Mary case, some understand the notion of "complete physical information" in such a way that it entails knowing everything about color perception, and hence they intuit that Mary does not learn something new upon her release (e.g., Dennett 1991), whereas others understand the notion of "complete physical information" in such a way that it does not entail knowing everything about color perception, and hence they intuit that Mary does learn something new upon her release (e.g., Jackson 1982). Likewise, in the violinist case, some might implicitly assume that they cannot afford to remain attached to the violinist for nine months, and hence they intuit that they are not obligated to, whereas others might implicitly assume that they can afford to remain attached to the violinist for nine months, and hence they

\footnotetext{
${ }^{8}$ Recall that both perceptions and intuitions probably vary across cultures. See footnotes 6 and 7 above.
} 
intuit that they are obligated to. Still others might not have any implicit assumptions about affordability at all.

Third objection. Some might insist that intuitions can still serve as prima facie, i.e., defeasible, evidence for premises in philosophical arguments, even in bad epistemic circumstances, such as in intuition pumps, because intellectual intuition affords one with some sort of direct access to the facts about hypothetical cases. In other words, an intellectual seeming (or intuitive appearance) that $p$ can still be a prima facie (i.e., defeasible) justification for $p$.

In reply to the third objection, I would like to make the following points. I think that there are several serious problems with the view of the evidential role of intuition according to which intuition gives $S$ direct epistemic access to the facts about hypothetical case $C$. Briefly, here are some of the most serious problems:

- If we take the analogy between intellectual intuition and sense perception seriously, then the direct-access view flies in the face of findings from perceptual psychology. As I have mentioned above, studies on illusions show that sense perception is expectation-laden (see, e.g., Deliza et al 2003). Such studies suggest that sense perception does not simply give us direct access (unmediated by inference, whether conscious or otherwise) to the facts. So, if sense perception and intellectual intuition are indeed analogous in epistemically relevant respects, then it is unlikely that intuition gives us direct access to the facts, either.

- If intuition gives $S$ direct access to the facts, how do we explain philosophical disagreement? Jackson intuits that Mary learns something new, but Dennett (1991) and others intuit that she does not. Do we want to say that Jackson has some special access to 
the facts that others do not? Indeed, how do we know that it is Jackson that has a special access to the facts about the Mary case, and not Dennett?

- If intuition gives $S$ direct access to the facts, how do we explain findings in experimental philosophy, which show that intuitions vary across cultures and are subject to order and framing effects? (See, e.g., Machery et al 2012.) If people simply intuit what is the case, why would cultural background make a difference? (Stich 1988)

Given these serious problems with the direct-access view of intuition, I submit, there are no good reasons to think that intellectual intuition gives us direct access to facts about hypothetical cases. Indeed, those who endorse the perception-intuition analogy, and who also wish to take empirical evidence seriously, should accept that intuition does not give us direct epistemic access to facts, given that perception probably doesn't.

Fourth objection. My argument, if sound, implies skepticism about perceptual judgments. But skepticism about perceptual judgments is unwarranted. Therefore, my argument must be unsound.

In reply to the fourth objection, I would like to make the following points. My argument does not imply skepticism about perceptual judgments. Rather, my argument implies skepticism about perceptual judgments that are made under bad epistemic circumstances, such as illusions. In the same way that we would not trust our perceptual judgments about the Müller-Lyer illusion or the Ponzo illusion, since illusions are bad epistemic circumstances, we should not trust intuitive judgments that are made under bad epistemic circumstances, such as the circumstances of intuition pumps. On the other hand, making perceptual judgments under good epistemic circumstances may still be a reliable way of fixing belief. Even making intuitive judgments 
under good epistemic circumstances might be a reliable way of fixing belief. But intuition pumps are not good epistemic circumstances, or so I argue.

Fifth objection. If my argument is sound, then it is self-defeating, for I have argued that the $\mathrm{MoC}$ is unreliable by using illusions as an intuition pump.

In reply to the firth, and final, objection, I would like to make the following points. At its core, my argument is not an appeal to intuition, as in Step 2 of the MoC. Rather, at its core, my argument is an argument by analogy. The analogy, as stated in Section 2, is the one between sense perception and intellectual intuition. If one accepts the perception-intuition analogy, as many do, then one must also accept that making judgments (whether perceptual or intuitive) under bad epistemic circumstances (such as illusions and intuition pumps) is not a reliable method of fixing belief. I take that to follow straightforwardly from the perception-intuition analogy. The question, then, is whether intuition pumps are bad epistemic circumstances. I argue that intuition pumps are bad epistemic circumstances because what we intuit in response to intuition pumps is not the facts about the hypothetical case in question but rather the details we fill in to make sense of that hypothetical case. The problem is that people fill in the details in different ways and there is no principled way to control for this kind of details-filling when constructing intuition pumps.

This is an interesting result, I submit, for the following reasons. First, the perceptionintuition analogy is usually invoked to support the use of intuitions as evidence in philosophical arguments, not argue against such a use (see, e.g., Hales 2012). Second, as the quotations in Section 1 illustrate, the MoC is a widely used method of philosophical argumentation. If it is indeed an unreliable method, as I have argued, then philosophers should stop using it. 


\section{Conclusion}

In this paper, I have argued that the method of cases (namely, the method of using intuitive judgments elicited by intuition pumps as evidence for and/or against philosophical theories) is not a reliable method of generating evidence for and/or against philosophical theories. In other words, the method of cases is not a reliable method of fixing philosophical belief. This is so because, if perception and intuition are analogous in epistemically relevant respects, then using intuition pumps to elicit intuitive judgments is like using illusions to elicit perceptual judgments. In both cases, judgments are made under bad epistemic circumstances.

\section{Acknowledgments}

A version of this paper was presented at the New Jersey Regional Philosophical Association conference in Bergen Community College (November 2012). I would like to thank the audience, especially Joshua Knobe, for their useful feedback. I am also grateful to David Morrow and an anonymous reviewer of Review of Philosophy and Psychology for helpful comments on earlier drafts.

\section{References}

Bach, K. (1984). Default Reasoning: Jumping to Conclusions and Knowing When to Think Twice. Pacific Philosophical Quarterly, 65, 37-58.

Bealer, G. (1998). Intuition and the Autonomy of Philosophy. In M. DePaul and W. Ramsey (Eds.), Rethinking Intuition: The Psychology of Intuition and Its Role in Philosophical Inquiry. Lanham, MD: Rowman and Littlefield.

Bealer, G. (2000). A Theory of the A Priori. Pacific Philosophical Quarterly, 81, 1-30. 
Bonjour, L. (1998). In Defense of Pure Reason: A Rationalist Account of A Priori Justification. Cambridge: Cambridge University Press.

Botvinick, M. and Cohen, J. (1998). Rubber hands 'feel' touch that eyes see. Nature, 391, 756.

Brendel, E. (2004). Intuition Pumps and the Proper Use of Thought Experiments. Dialectica, 58, $88-108$.

Brogaard, B. (forthcoming). Intuitions as Intellectual Seemings. In D. Sosa (Ed.), Symposium on Herman Cappelen's Philosophy without Intuitions. Analytic Philosophy.

Buckwalter, W. (2012). Surveying Philosophers: A Reply to Kuntz and Kuntz. Review of Philosophy and Psychology, 3, 515-524.

Cappelen, H. (2012). Philosophy without Intuitions. New York: Oxford University Press.

Chudnoff, E. (2011a). The Nature of Intuitive Justification. Philosophical Studies, 153, 313-333.

Chudnoff, E. (2011b). What Intuitions Are Like? Philosophy and Phenomenological Research, $82,625-654$.

Chudnoff, E. (2013). Intuitive Knowledge. Philosophical Studies, 162, 359-378.

Coren, S. and Girgus, J. A. (1978). Seeing Is Deceiving: The Psychology of Visual Illusions. Hillsdale, NJ: Erlbaum.

Deliza, R., MacFie, J. H., and Hedderley, D. (2003). Use of Computer-Generated Images and Conjoint Analysis to Investigate Sensory Expectations. Journal of Sensory Studies, 18, 465-486.

Dennett, D. C. (1984). Elbow Room: The Varieties of Free Will Worth Wanting. MIT Press. 
Dennett, D. C. (1991). Consciousness Explained. Boston: Little Brown.

Dennett, D. C. (1995). Intuition Pumps. In J. Brockman (ed.), The Third Culture: Beyond the Scientific Revolution (pp. 180-197). New York: Simon \& Schuster.

Dorbolo, J. (2006). Intuition Pumps. Minds and Machines, 16, 81-86.

Ehrsson, H. H., Holmes, N. P., and Passingham, R. E. (2005). Touching a rubber hand: Feeling of body ownership is associated with activity in multisensory brain areas. Journal of Neuroscience, 25, 10564-10573.

Goldman, A. I. (2007). Philosophical Intuitions: Their Target, Their Source, and Their Epistemic Status. Grazer Philosophische Studien, 74, 1-26.

Hales, S. D. (2012). The Faculty of Intuition. Analytic Philosophy, 53, 180-207.

Huemer, M. (2007). Compassionate Phenomenal Conservatism. Philosophy and Phenomenological Research, 74, 30-55.

Jackson, F. (1982). Epiphenomenal Qualia. Philosophical Quarterly, 32, 127-136.

Kornblith, H. (2007). Naturalism and Intuitions. Grazer Philosophische Studien, 74, 27-49.

Kuntz, J. R. and Kuntz, J. R. C. (2011). Surveying Philosophers about Philosophical Intuition. Review of Philosophy and Psychology, 4, 643-665.

Lewkowicz, D. J. and Ghazanfar, A. (2009). The emergence of multisensory systems through perceptual narrowing. Trends in Cognitive Science, 13, 470-478.

Ludwig, K. (2010). Intuitions and relativity. Philosophical Psychology, 23, 427-445. 
Machery, E., Mallon, R., Nichols, S., and Stich, S. (2012). If Folk Intuitions Vary, Then What?

Philosophy and Phenomenological Research. DOI: 10.1111/j.1933-1592.2011.00555.x.

Maudlin, T. (2007). The Metaphysics within Physics. New York: Oxford University Press.

McGurk, H. and MacDonald, J. (1976). Hearing lips and seeing voices. Nature, 264, 746-748.

Mizrahi, M. (2012). Intuition Mongering. The Reasoner, 6, 169-170.

Mizrahi, M. (2013). More Intuition Mongering. The Reasoner, 7, 5-6.

Nagel, J. (2012). Intuitions and Experiments: A Defense of the Case Method in Epistemology. Philosophy and Phenomenological Research, 85, 495-527.

Pryor, J. (2005). There is Immediate Justification. In M. Steup and E. Sosa (Eds.), Contemporary Debates in Epistemology. Malden, MA: Blackwell.

Pust, J. (2001). Against Explanationist Skepticism Regarding Philosophical Intuitions.

Philosophical Studies, 106, 227-258.

Rescher, N. (2005). What if? Thought Experimentation in Philosophy. New Brunswick, NJ:

Transaction Publishers.

Rookes, P. and Willson, J. (2000). Perception: Theory, Development and Organisation. London: Routledge.

Schett, A. (1999). The discovery of the ophthalmoscope by Hermann von Helmholtz. Strabismus, 7, 241-144. 
Segall, M. H., Campbell, D. T., and Herskovits, M. J. (1963). Cultural Differences in the Perception of Geometric Illusions. Science, 139, 796-771.

Slater, M., Spanlang, B., Sanchez-Vives, M. V., Blanke, O. (2010). First Person Experience of Body Transfer in Virtual Reality. PLoS ONE 5(5): e10564. doi:10.1371/journal.pone.0010564.

Sosa, E. (1996). Rational Intuition: Bealer on Its Nature and Epistemic Status. Philosophical Studies, 81, 151-162.

Sosa, E. (2007). Experimental Philosophy and Philosophical Intuition. Philosophical Studies, 132, 99-107.

Sosa, E. (2009). Replies to Commentators on a Virtue Epistemology (Oxford University Press, 2007). Philosophical Studies, 144, 137-147.

Stein, B. E. and Meredith, M. A. (1993). The Merging of the Senses. Cambridge, MA: MIT Press.

Stich, S. (1988). Reflective Equilibrium, Analytic Epistemology and the Problem of Cognitive Diversity. Synthese, 74, 391-413.

Stich, S. (2012). Do Different Groups Have Different Epistemic Intuitions? A Reply to Jennifer Nagel. Philosophy and Phenomenological Research. DOI: 10.1111/j.1933-1592.2012.00590.x.

Swain, S., Alexander, J., \& Weinberg, J. M. (2008). The instability of philosophical intuitions:

Running hot and cold on Truetemp. Philosophy and Phenomenological Research, 76, 138-155.

Thomson, J. J. (1971). A Defense of Abortion. Philosophy and Public Affairs, 1, 47-66.

Williamson, T. (2004). Philosophical 'Intuitions' and Skepticism about Judgement. Dialectica, $58,109-153$. 
Williamson, T. (2007). The Philosophy of Philosophy. New York: Wiley-Blackwell. 